\title{
EL JOVEN DIOS DEL MAÍZ. \\ TECNOLOGÍA Y SIMBOLISMO DE UN PENDIENTE DE CONCHA DEL MUSEO NACIONAL DE ANTROPOLOGÍA
}

THE YOUNG GOD OF MAIZE. TECHNOLOGY AND SYMBOLISM OF A SHELL PENDANT

FROM THE NATIONAL MUSEUM OF ANTHROPOLOGY

\author{
Daniel JuÁrez Cossío \\ Museo Nacional de Antropología, \\ Instituto Nacional de Antropología e Historia \\ Adrián Velázquez Castro \\ Museo del Templo Mayor, \\ Instituto Nacional de Antropología e Historia \\ Norma Valentín Maldonado \\ Subdirección de Laboratorios y Apoyo Académico, \\ Dirección de Estudios Arqueológicos, \\ Instituto Nacional de Antropología e Historia
}

\begin{abstract}
Resumen: En el área maya se han reportado numerosos objetos elaborados en concha de caracol que representan individuos en escenas cortesanas o bien divinidades en pasajes mitológicos. Estas piezas, exquisitamente trabajadas, muestran la silueta recortada y calada del personaje, detallándose su representación mediante líneas incisas y en ocasiones diseños excavados para incluir incrustaciones. Se ha planteado la posibilidad de que un buen número de ellas corresponda a un mismo estilo de manufactura.

La gran mayoría de las piezas pertenece a colecciones privadas, otras en cambio carecen de contexto arqueológico. La colección maya del Museo Nacional de Antropología de México cuenta con un único ejemplar de este tipo, conocido como "El fumador", procedente de las excavaciones realizadas en Jaina en 1964. Es propósito del presente trabajo exponer los resultados del análisis iconográfico y tecnológico de esta pieza, para discutir su pertenencia a dicha tradición manufacturera.
\end{abstract}

Palabras clave: Jaina, Clásico Tardío, Dios del maíz, ornamentos de concha, técnicas de manufactura.

ABSTRACT: A number of pieces made from seashells representing individuals in courtly settings or divinities in mythological scenes have been reported in the Maya area. These exquisitely worked artifacts display the openwork cutout silhouette of the figure, with details rendered by incised lines and carved out areas to inset inlays. It has been proposed that a number of these objects belong to the same manufacturing style. Most of the pieces are in private collections and lack archaeological con- 
text. The Maya collections in the National Museum of Anthropology in Mexico City have a unique example of this type that comes from an excavated context. Known as "The Smoker," it was found at the island of Jaina in 1964. This paper presents the results of iconographic and technological analyses of this important artifact, in order to explore its relationships to the above-mentioned tradition.

KEYworDS: Jaina, Late Classic, Maize god, seashell ornaments, manufacturing techniques.

RECEPCIÓn: 5 de febrero de 2015.

ACEPTACIÓN: 29 de abril de 2015. 


\title{
EL JOVEN DIOS DEL MAÍZ \\ TECNOLOGÍA Y SIMBOLISMO \\ DE UN PENDIENTE DE CONCHA DEL MUSEO \\ NACIONAL DE ANTROPOLOGÍA ${ }^{1}$
}

\author{
Daniel JuÁrez Cossío \\ Museo Nacional de Antropología, \\ Instituto Nacional de Antropología e Historia \\ Adrián Velázquez Castro \\ Museo del Templo Mayor, \\ Instituto Nacional de Antropología e Historia \\ Norma Valentín Maldonado \\ Subdirección de Laboratorios y Apoyo Académico, \\ Dirección de Estudios Arqueológicos, \\ Instituto Nacional de Antropología e Historia
}

El mito es una norma, el esquema de un hecho acaecido de una vez por todas, y extrae su valor de esa unicidad absoluta que lo conduce fuera del tiempo y lo consagra como una revelación.

Cesare Pavese, El oficio de vivir.

Para el Mtro. Roberto Jiménez Ovando (1928-2013), quien generosamente compartió con nosotros el recuerdo de sus excavaciones en Jaina.

La Sala Maya del Museo Nacional de Antropología exhibe una excepcional pieza elaborada sobre una placa de concha procedente de la isla de Jaina. En ella se representó al joven Dios del maíz acompañado por un ave acuática, cuyo pico está en contacto con la nariz de la deidad. Este rasgo particular llevó a diversos investigadores a identificar al personaje como un fumador, acaso un gobernante practicando algún ritual.

\footnotetext{
${ }^{1}$ Una versión preliminar de este trabajo se presentó en el IX Congreso Internacional de Mayistas, celebrado en la ciudad de San Francisco de Campeche, Campeche, entre los días 23 al 29 de junio del 2013.
} 
En este trabajo abordaremos el estudio del pendiente, incluyendo las técnicas de su manufactura, ya que son relativamente pocas las piezas elaboradas en concha con las cuales comparte algunos rasgos. Un segundo aspecto que nos interesa tratar es el de la representación simbólica, que lo identifica como el joven Dios del maíz, cuya iconografía figura, fundamentalmente, en vasijas policromadas. $^{2}$

Desafortunadamente, muchas de las piezas procedentes de Jaina y que forman parte de la colección del museo no cuentan con registros respecto a sus relaciones contextuales, lo que limita sensiblemente su comprensión con referencia al sistema simbólico dentro del cual participaron. Tal es el caso del pendiente que nos ocupa, la cual, a pesar de proceder de un proyecto de investigación, tampoco cuenta con el registro que permita comprender el contexto de su hallazgo. Es por ello que nos limitaremos a tratar sólo su aspecto tecnológico e iconográfico.

\section{La isla de Jaina: historia en migajas}

En 1964 Román Piña Chan y Luis Aveleyra fueron comisionados para realizar exploraciones en Jaina. El objetivo central de dichos trabajos estuvo encaminado a incrementar el acervo de las colecciones mayas del Museo Nacional de Antropología, que por aquel entonces se construía en el Bosque de Chapultepec y cuyo secretario de Planeación era el propio Aveleyra. ${ }^{3}$

No era la primera vez que los trabajos de exploración tenían como propósito la obtención de piezas para su exhibición. Piña Chan señaló en su obra Jaina, La casa en el agua (1968) que la expedición de 1947, en la que también colaboró Pavón, tuvo el mismo objetivo. Sólo que por aquel entonces la colecta de materiales se destinó a la reestructuración de la Sala Maya del antiguo Museo alojado en la calle de Moneda. Por ello resulta paradójico lo asentado en tal publicación: “[...] con los fondos necesarios y con la experiencia de las exploraciones pasadas, pudo planearse ella [la expedición] en todos sus detalles, lo cual aseguró el éxito de los trabajos" (Piña Chan, op. cit.: 14). Estas palabras contrastan de manera sensible con lo que páginas más adelante refiere el autor al describir las características arquitectónicas de los conjuntos Zayosal y Zacpool, que además fueron saqueados, pues tal situación, aunada a las pocas excavaciones sistemáticas que se habían realizado, prácticamente imposibilitaban el estudio del asentamiento: "En realidad, casi nada se sabe de la arquitectura y técnicas constructivas del centro ceremonial, por lo cual es necesario realizar exploraciones intensivas del lu-

\footnotetext{
${ }^{2}$ A decir de Erik Velásquez (2010), durante el período Clásico, el Dios del maíz era conocido con diversos nombres, siendo quizá el de Ju’n Ixiim o 1 Maíz el que mejor lo describe.

${ }^{3}$ En las excavaciones participaron Raúl Pavón, Agustín Delgado y Héctor Gálvez, quienes contaron con la colaboración de los antropólogos físicos Roberto Jiménez Ovando y Sergio López Alonso, además de los pasantes de arqueología Hilda Schmidt de Delgado y Guacolda Boisset. El estudio de la cerámica fue realizado por los entonces estudiantes Yoko Sugiura y Juan Pedro Laporte.
} 
gar, pero es casi seguro que hay subestructuras y construcciones tempranas, que explicarían el crecimiento y evolución de dicho centro" (Piña Chan, op. cit.: 35).

De tal manera que tras el subjuntivo "explicarían" de la cita anterior, bien podemos reducir "el éxito de los trabajos" a la enorme cantidad de piezas que se obtuvo para el museo. En efecto, una parte significativa de los materiales que conforman el acervo de la colección maya son precisamente de Jaina, y en su mayoría corresponden a figurillas que estuvieron incorporadas a contextos funerarios. Pese a ello, conocemos casi nada sobre la relación contextual entre unidades arquitectónicas, entierros y ofrendas. Por otra parte, con los resultados derivados del análisis de las figurillas se lograron establecer cinco tipos; cuatro quedaron comprendidos entre los años 300 y 650 d.C. y corresponden a la ocupación temprana de la isla, y el último tipo entre los años 650 y 1000 d.C. En el texto de Piña Chan también se advierte que no hay correlación alguna entre las figurillas y la cerámica a la cual se asocian, de tal manera que desconocemos los criterios empleados para su datación. Sólo sabemos que éstas formaban parte de ofrendas funerarias, las cuales por lo general se colocaban entre los brazos de los individuos con otros artefactos elaborados en piedra verde, hueso y concha. ${ }^{4}$

Si esto ocurrió con las figurillas que por la delicadeza de su manufactura constituyen un material sumamente apreciado, en especial por los coleccionistas, comprenderemos la poca atención que recibieron otro tipo de artefactos como los elaborados en concha, plenos de información que, por aquellos años, nadie imaginaba. Esto último no sólo desde la perspectiva elemental que supone la identificación de especies y su provincia biótica (lo que permite esbozar la manera en que interactuaron con otros sitios y regiones), sino también las implicaciones que esto conlleva respecto a sus formas de obtención, distribución y consumo. Destaca también el avance alcanzado durante la última década en cuanto al conocimiento sobre técnicas de manufactura, mediante las cuales fue elaborado este tipo de artefactos, así como la cadena operativa dentro de la cual se insertó su producción.

A las consideraciones anteriores quizá debamos agregar, dentro del campo de la representación simbólica, su naturaleza acuática, ya que su transformación en objetos para el consumo de las élites les confirió un carácter significativo. Resulta interesante en este sentido el argumento expuesto por Stephen D. Houston (2010: 78) sobre la posibilidad de que las conchas hubiesen personificado el

\footnotetext{
${ }^{4}$ En 2005 Serrano Sánchez y López Alonso (2007: 95) participaron en un simposio dedicado a la población prehispánica de Jaina en el marco del XIII Coloquio Juan Comas. En él reelaboraron algunos de los datos obtenidos durante sus trabajos de excavación entre 1973 y 1974, e hicieron observaciones interesantes en cuanto a patrones funerarios. Entre otras prácticas destacó la preparación del cuerpo mediante la aplicación de pintura roja o amarilla, la colocación de una o varias figurillas entre los brazos y la de una cuenta de piedra verde dentro de la boca. Los cuerpos fueron depositados de manera flexionada y dispuestos directamente sobre la fosa donde probablemente se quemó copal. Los niños fueron colocados dentro de vasijas tapadas con un cajete, y las mujeres, aparentemente, no gozaron de un particular reconocimiento tras la muerte. Sobra decir que la calidad de las ofrendas variaba de acuerdo al rango social al que pertenecía el individuo.
} 
aliento vital de los ancestros, quizá porque el agua de donde proceden constituye un espacio liminar. De tal manera que podríamos considerar que algunas de estas piezas, más que como ornamentos, fueron percibidas como puentes de comunicación con los ancestros. Más aún, bajo esta perspectiva, también tenemos la posibilidad de estudiar el entramado de vínculos sociales que se construyeron mediante la redistribución y el intercambio de presentes, los cuales, evidentemente, establecieron formas de comunión, a las que Arnold Van Gennep (2008: 53) definió como "ritos de agregación material".

\section{"El fumador": sus espirales son sueños celestiales...}

De entre los numerosos artefactos manufacturados sobre concha que fueron recuperados en Jaina, uno de los más conocidos es el pendiente que muestra el torso de un personaje. Originalmente fue publicado por Piña Chan en 1968 (1968: 72) (figura 1) y se ilustra como parte de la lámina 15 de su publicación junto con otros interesantes artefactos también de concha, en especial los dos primeros, que seguramente fueron utilizados como tinteros por escribas. En el capítulo "Los artefactos y obras artesanales", Piña Chan dedica apenas tres párrafos al material conquiliológico, los cuales incluyen descripciones muy generales. Conforme al registro inscrito en la pieza, ésta procede de la sección D, trinchera 1, y corresponde a una "pieza aislada” a la que se le asignó el número 606. Serrano Sánchez y López Alonso

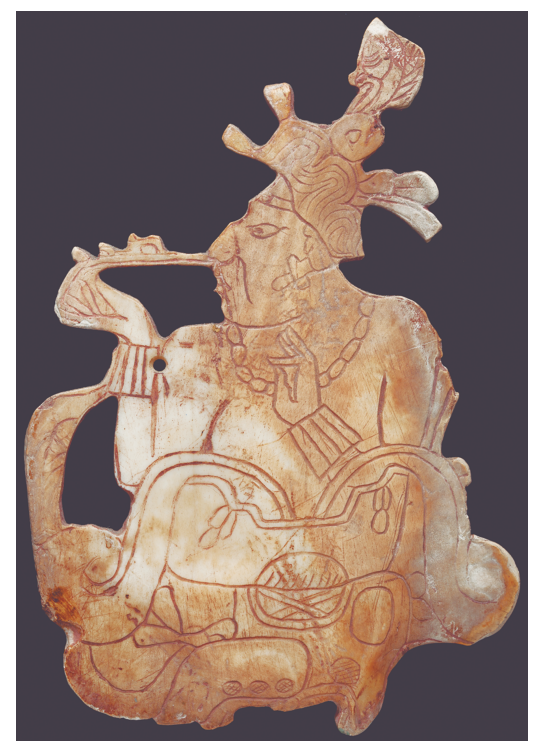

Figura 1. Anverso del pendiente de concha que muestra al Dios del maíz. Museo Nacional de Antropología, No. de catálogo 05.0-01391. Fotografía: Archivo Digital. 
(2007: 82) advirtieron que las figurillas resultaron ser el ajuar más recurrente en el enterramiento de hombres y niños, pero no en el caso de las mujeres. Cuentas, orejeras y anillos fueron colocados indistintamente en ambos sexos, a excepción de los collares, más comunes entre los infantes. Las conchas sin modificación alguna se asocian a las mujeres, mientras que las placas de concha a los hombres. Resulta posible suponer entonces que dicha placa debió formar parte del ajuar de un personaje que ocupó cierto rango destacado dentro de su comunidad.

En opinión de Antonio Benavides (com. pers., 2013), quien realizó excavaciones en Jaina entre 1996 y 2005, la pieza pudo haber sido recuperada en un sector habitacional situado en la porción septentrional de la isla, ya que en este lugar se practicaron diversas trincheras a poco menos de $100 \mathrm{~m}$ al norte de la cancha del Juego de Pelota. Benavides nos hizo notar que el entierro 24, ilustrado en la figura 31 de la citada publicación, se localizó precisamente en esa sección.

La pieza fue trabajada sobre un trozo de la última involución de la espira de un caracol Strombus gigas, lo cual resulta evidente en la cara posterior, donde se aprecia la ornamentación natural característica de la especie, consistente en costillas espirales bien marcadas que se abren hacia el labio externo (figura 2). Cabe recordar que la especie Strombus gigas se distribuye desde Tamaulipas hasta el Mar Caribe (García Cubas y Reguero, 2004: 83), habita en sustratos arenosos donde crecen pastos marinos de los cuales se alimenta (Frenkiel y Aldana, 2003: 64-65) y ha sido un molusco muy apreciado por su carne, en tanto que su concha se usó en muchos sitios y épocas de Mesoamérica para la elaboración de objetos suntuarios y rituales.

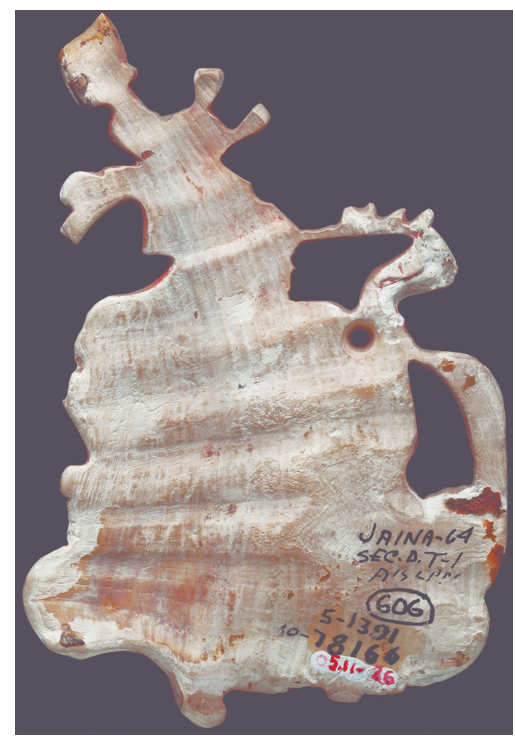

FIGURA 2. Reverso del pendiente de concha donde se observan las costillas espirales del Strombus. Museo Nacional de Antropología, No. de catálogo 05.0-01391. Fotografía: Archivo Digital. 
En la pieza que nos ocupa se representó el torso del joven Dios del maíz vuelto hacia su costado derecho; brota de una montaña hendida o witz en plena floración. La montaña fue personificada mediante un ojo con pupila de bandas cruzadas que asoma bajo el párpado estriado, generalmente asociada con el lagarto terrestre. Benavides (2012: 229), apoyado en García Campillo (1998), sugiere que las bandas cruzadas podrían hacer referencia al emblema jeroglífico de Jaina; sin embargo, Erik Velásquez (com. pers., 2013) no advierte grafema alguno de escritura en la pieza. Uno de los rasgos más interesantes lo constituye la silueta de un cormorán, una garza o algún otro tipo de ave acuática, elemento que Houston (op. cit.) identificó como una emanación que exhala de la nariz. Es por ello que a esta pieza se le denominó, de forma inadecuada, como "El fumador".

La silueta del personaje se consiguió perfilar mediante recortes elaborados con gran destreza, incluyendo dos finos calados que separan a la divinidad del cormorán. El resto de los detalles se obtuvo mediante líneas incisas trazadas con gran maestría sobre la superficie interna de la concha, mientras que la parte externa fue desgastada para eliminar al máximo las irregularidades propias del material. Se aprecia una perforación bicónica donde convergen el hombro derecho y la muñeca del personaje. También se advierte otra horadación a la misma altura pero en el brazo izquierdo, la cual bien pudo haberse fracturado durante su elaboración, ya que el defecto trató de atenuarse desgastando la superficie.

Uno de los detalles que llama la atención respecto a la manufactura de la pieza es que, para la elaboración de los recortes y los calados, se usaron perforaciones que, o bien suavizan ángulos, o bien se hicieron de forma contigua para posteriormente unirse mediante desgaste.

El análisis sobre técnicas de manufactura fue realizado en el marco del proyecto "Técnicas de Manufactura de los Objetos de Concha del México Prehispánico" (PTMOCMP), que se desarrolla en el Museo del Templo Mayor. En él, y considerando la frecuente carencia de indicadores directos de la producción, ${ }^{5}$ los aspectos tecnológicos son estudiados bajo el esquema de la arqueología experimental. Esto tiene como propósito replicar en conchas modernas, de las mismas especies empleadas en la época prehispánica, las modificaciones realizadas en la antiguiedad para manufacturar los objetos conquiliológicos. Para ello se utilizan los materiales e instrumentos que, por diferentes fuentes de información, ${ }^{6}$ suponemos que se emplearon en el pasado.

Para rebasar el nivel estrictamente hipotético y con el propósito de aproximarnos con mayor seguridad a los procedimientos y utensilios empleados, las huellas de manufactura experimentales se caracterizan y se comparan con los rasgos presen-

\footnotetext{
${ }^{5}$ Es muy frecuente que los objetos de concha se localicen en contextos de ofrenda, por lo que se hallan piezas terminadas. Los indicadores directos de la producción (piezas falladas e instrumentos agotados) se encuentran usualmente en contextos secundarios, como basureros o rellenos constructivos, y en ocasiones en los lugares donde se llevaba a cabo la elaboración de los objetos.

${ }^{6}$ Las fuentes de información son los hallazgos arqueológicos, los documentos históricos y las propuestas de otros investigadores.
} 
tes en las piezas arqueológicas. Esto se realiza bajo tres niveles de análisis: macroscópico (simple vista), microscopía estereoscópica de baja amplificación (10x, 30x y 63x) y microscopía electrónica de barrido (MEB) (100x, 300x, 600x y 1000x). Esta última técnica ha resultado ser la más eficiente para determinar las características presentes en la superficie de los materiales y la hemos descrito ampliamente en otros reportes (Velázquez, 2007; Velázquez y Juárez, 2007).

En un inicio el estudio de los objetos de concha con MEB se hacía trasladando las piezas al laboratorio; después se optó por obtener réplicas de las huellas de manufactura en polímeros reblandecidos con acetona, técnica propia de la metalografía, los cuales son recubiertos con iones de oro. Entre otras ventajas, ello evita el traslado de las colecciones arqueológicas, dado que dichas muestras pueden tomarse en sus repositorios. De la misma forma hace posible la observación en modo de alto vacío, lo cual permite un mayor nivel de resolución, ya que la presencia de aire dentro de la cámara de muestreo no permite la libre trayectoria de los electrones desde el material hasta los detectores del microscopio, pues produce una carga electrostática que imposibilita observar con nitidez los rasgos de los materiales (Velázquez, 2007).

Para el análisis se obtuvieron cinco polímeros de la pieza de Jaina, que incluyeron las superficies anterior y posterior, bordes e incisiones. ${ }^{7}$ Dichas réplicas fueron recubiertas con iones de oro y observadas en modo de alto vacío, con una potencia del haz de $20 \mathrm{kV}$ en apertura de 42, con señal de electrones secundarios (SEI) y a una distancia de trabajo de $10 \mathrm{~mm}$, lo que permitió hacer registros micrográficos de hasta cuatro amplificaciones cada uno de ellos (100x, 300x, 600x y 1000x). Posteriormente, las micrografías se analizaron y compararon con la amplia base de datos del PTMOCMP. En lo que respecta a las superficies, se observan rasgos de tres diferentes medidas: líneas de $5.8 \mu \mathrm{m}$ y bandas de $23 \mathrm{y}$ $58 \mu \mathrm{m}$, lo cual se corresponde con las huellas que produce el desgaste con rocas calizas (figura 3). En los bordes y las líneas incisas se aprecian finas líneas rectas de $0.6 \mu \mathrm{m}$ de ancho, similares a las que deja el empleo de instrumentos afilados de obsidiana (figura 4).

\section{Piezas similares}

En 2012 se publicó el catálogo Ancient Maya Art at Dumbarton Oaks, editado por Pillsbury et al., donde se presentan, entre muchos otros objetos, seis ornamentos elaborados en concha que forman parte de los acervos de aquel museo. El estudio sobre las técnicas de manufactura fue realizado por Velázquez Castro; mientras que los aspectos iconográficos y epigráficos fueron tratados por Houston. El catálogo también incluye información sobre las circunstancias de su adquisición y las expo-

\footnotetext{
${ }^{7}$ Vale la pena mencionar que se tomaron polímeros de algunas de las perforaciones, en las cuales no se pudieron observar las huellas de manufactura debido al deterioro de la pieza.
} 
siciones en las que han participado, así como las referencias bibliográficas donde se les menciona.
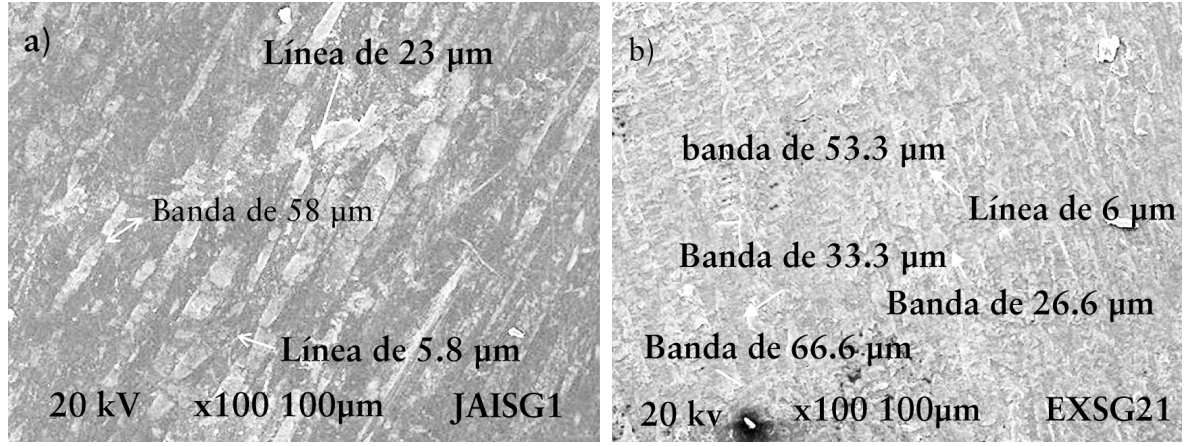

Figura 3. Micrografías MEB de a) superficie del pendiente arqueológico y b) superficie de Strombus gigas desgastada experimentalmente con caliza, ambas a 100x. Micrografías: PTMOCMP.
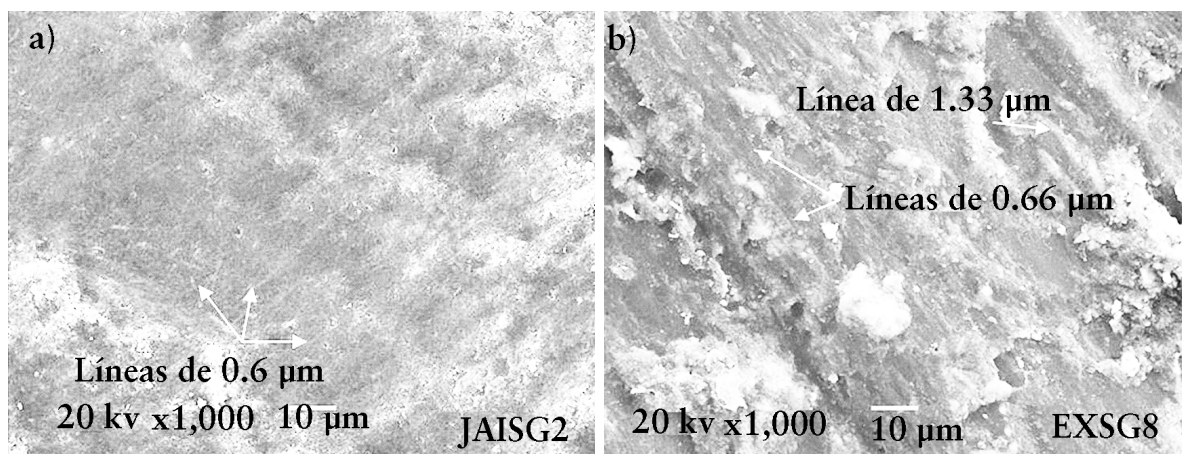

FiguRA 4. Micrografías MEB de a) borde del pendiente arqueológico y c) corte experimental con lascas de obsidiana en Strombus gigas, ambas a 1000x. Micrografías: PTMOCMP.

De este conjunto de ornamentos nos interesa destacar el pendiente registrado como PC.B.543 y de procedencia desconocida. Es también una representación del joven Dios del maíz vuelto hacia su lado derecho y surgiendo del enorme ojo del monstruo xook con pupila de bandas cruzadas, muy similar al que aparece representado en el vaso K1391. En su descripción, Houston señala que este pez fantástico también fue representado en los cinturones reales afianzando una concha de Spondylus entre sus fauces. Taylor (1992), por su parte, señala que este tipo de cinturones se relaciona con ritos de sangre y autosacrificio, utilizados generalmente con un faldellín tejido como red con cuentas.

Resulta interesante destacar las similitudes en cuanto a técnicas de manufactura que tiene este objeto con el de Jaina. También se elaboró a partir de un 
fragmento de Strombus gigas, donde la silueta del monstruo y la divinidad se recortaron con preciosismo. Algunos rasgos fueron resaltados mediante calados utilizando perforaciones sucesivas. Finalmente, los detalles de las representaciones se lograron trazando líneas incisas hechas con gran maestría. La única diferencia significativa consiste en pequeñas zonas excavadas en el objeto de Dumbarton Oaks, las cuales se practicaron con toda seguridad para incrustar materiales como jadeíta, Spondylus y hematita (Velázquez, 2012).

Conocemos dos pendientes más elaborados en concha que muestran los mismos rasgos iconográficos del joven Dios del maíz. Uno procede de las excavaciones de Xcambó, en Yucatán, y actualmente forma parte del acervo del Museo Regional de Yucatán en el Palacio Cantón. Sierra (2004) lo sitúa dentro del Complejo Xcambó del Clásico Tardío, entre los años 650 y 750 d.C., y lo describe como un noble sentado sobre un trono zoomorfo bicéfalo. La cabeza izquierda podría representar un cocodrilo celeste y la otra la del monstruo del lirio acuático. El cuerpo muestra espirales identificadas como las bandas de la tierra (figura 5).

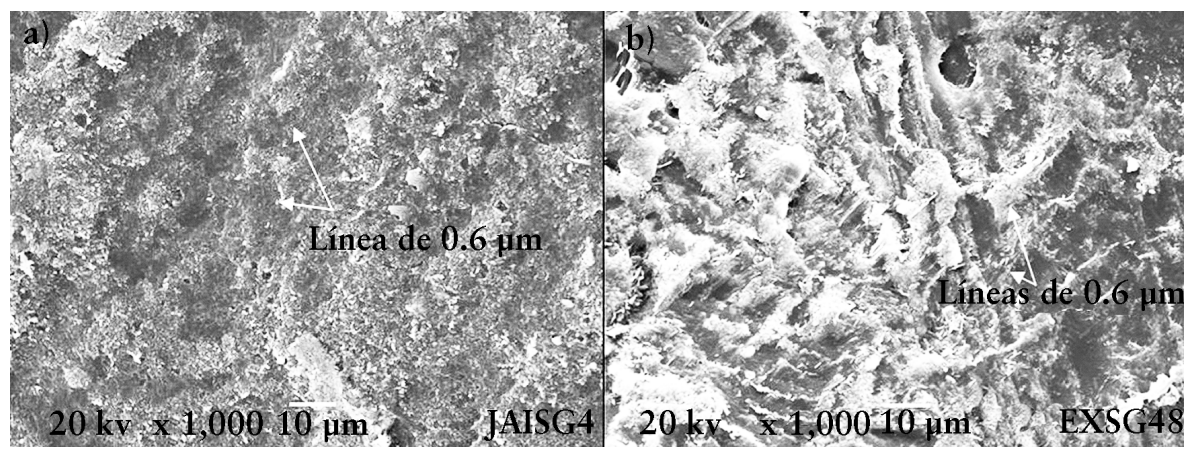

FIGURA 5. Micrografías MEB de a) incisión del pendiente arqueológico y b) incisión hecha experimentalmente con lascas de obsidiana en Strombus gigas, ambas a 1000x. Micrografías: PTMOCMP.

El otro pendiente proviene de las exploraciones en Copán, Honduras, forma parte de los acervos del Museo Regional de Arqueología de aquella entidad con el número de inventario CPN: CPN-H-77, y aparece en la ficha 289 del catálogo de la exposición Los Mayas (Schmidt, Garza y Nalda, 1998: 590). William L. Fash menciona que esta pieza, localizada en un conjunto habitacional a menos de $200 \mathrm{~m}$ al norte de la Gran Plaza de Copán, podría ser la representación de un ancestro. En otras publicaciones hace notar que este conjunto fue explorado por Núñez Chinchilla (Fash, 1991: 57; Fash, 1996: 42). De acuerdo con esta información, resulta muy probable que provenga del Grupo 9-L-23. ${ }^{8}$

\footnotetext{
${ }^{8}$ La ubicación y configuración de este Grupo se puede observar en el Mapa 12, Grupo Principal, publicado en el tomo III del Proyecto Arqueológico Copán, dirigido por Baudez en 1978 (Baudez, 1983).
} 
La pieza representa la cabeza de un reptil fantástico con cuerpo en forma de lirio acuático. Al centro destaca la silueta del joven Dios del maíz mirando hacia la derecha y rodeado por incrustaciones de jadeíta y concha. Conviene agregar, finalmente, que estos dos últimos pendientes no se han estudiado desde la perspectiva de su técnica de manufactura y tampoco conocemos la especie taxonómica a la cual pertenecen.

\section{El programa iconográfico de Ju'n Imiix, el Dios de maíz}

El tema central en el pendiente de concha que nos ocupa, al igual que en los otros dos referidos, es el nacimiento del Dios del maíz, cuyo programa iconográfico es amplio y complejo. En su representación se incorporan elementos de la cosmovisión maya que permearon el imaginario del mundo Clásico, como los cerros personificados, cuya vegetación simboliza la fecundidad y abundancia, así como el monstruo xook, el lagarto o bien la tortuga, como representación de la superficie terrestre flotando sobre las aguas primordiales.

Conviene indicar que abordaremos esta temática desde la perspectiva de la Antropología Simbólica y tratando de acotarla desde su propio campo semántico. Para ello hemos considerado que los referentes básicos constituyen símbolos, a los cuales se les dotó de un significado que fue compartido por las comunidades dentro de los cuales participaron instrumentalmente. En modo alguno pretendemos establecer la narrativa construida alrededor del mito Dios del maíz en el imaginario del mundo maya. Es probable que ésta haya asumido variantes e incorporado a lo largo del tiempo algunos otros elementos.

En el itinerario que nos hemos trazado, esbozamos la distinción propuesta por Paul Ricœur entre mensaje y código. El primero constituye un acontecimiento temporal en su dimensión diacrónica. Por su parte, el código configura elementos en un sistema sincrónico: "El mensaje es intencional [...]; El código es anónimo y no intencionado" (Ricœur 1998:17). En consecuencia, el pendiente que nos ocupa forma sólo una parte del mensaje cuyo discurso como acontecimiento debió ser conocido durante el Clásico Tardío en la costa campechana: el ciclo del Dios del maíz. Bajo esta premisa, la secuencia que proponemos se nutre de las referencias iconográficas mejor conocidas y consensuadas en el imaginario de la constructio académica. De igual forma nos hemos apoyado en referencias etnográficas y asumimos, como apunta Sahlins, que "en la sociedad humana, ningún objeto o cosa tiene existencia ni movimiento salvo por el significado que los hombres puedan asignarle" (2006: 170).

Karl Taube (1985: 172) señaló que el Dios del maíz fue identificado desde finales del siglo xix por Schellhas como Dios E, debido a su cabeza en forma de mazorca con foliaciones. Apuntó también que hacia principios del siglo pasado, los estudios de Seler, Dieseldorff y Spinden (1975: 89) coincidieron y consensuaron su identificación. El avance en el campo de la epigrafía, que en la década de los 
años setenta del siglo pasado marcó un parteaguas en la investigación arqueológica, ha sido un apoyo importante para entender el rico y complejo programa iconográfico que gravita alrededor de esta deidad.

La alegoría del ciclo agrícola nos ha sido revelada a través de escenas que quedaron plasmadas en vasijas del período Clásico y, en menor escala, en ornamentos como el que nos ocupa. Las escenas que aquellos artistas eligieron como "texto" por lo común no establecen una narración lineal de los eventos míticos relatados; describen sólo partes de la saga, lo que dificulta hilvanar la secuencia de la trama. A esto debiéramos agregar quizá las variaciones regionales, que seguramente introdujeron modificaciones o adaptaciones, como, por lo general, ocurre entre los narradores.

Sin embargo, y a pesar de tales variantes, las escenas muestran al Dios del maíz viajando hacia el inframundo en una canoa impulsada por los Dioses remeros como metáfora de su muerte ritual. También se le representó en aquel ámbito oscuro dominado por el Dios L, con quien mantiene una ríspida confrontación de la que sale vencedor. Como preludio al trance que está por ocurrir, departe con un alegre grupo de doncellas que juguetean desnudas dentro del agua mientras lo atavían como danzante. Sobreviene entonces la eclosión del caparazón, símbolo de la superficie terrestre, para dar paso al nacimiento de la deidad que brota como una planta. El ciclo culmina con una danza ritual para proclamar su triunfo sobre la muerte. El Dios del maíz baila con un tocado de lirios y plumas de quetzal ante la mirada de los Dioses con diadema (Dioses Ch y S), acompañado por una garza o cormorán en cuyo pico se agita un pescado. Estas son, a grandes rasgos, las escenas que se han identificado. ${ }^{9}$

Chinchilla (2011: 53) describe e interpreta tres escenas representadas en el Vaso de los Remeros que pertenece al acervo del Museo Popol Vuh en Guatemala: las mujeres desnudas que visten al Dios del maíz; su viaje en la canoa conducida por los Dioses remeros; y finalmente su alumbramiento entre las fauces de una serpiente acuática. Asume que no es una narración completa y que acaso el artista recurrió a pasajes de la versión que circulaba en el noreste del Petén durante el Clásico Tardío, opinión que compartimos. En la secuencia que se advierte entre líneas, trazada por Chinchilla, la saga sigue la misma secuencia que hilvanó para describir el vaso. Es decir, inicia con el atavío y baile del Dios del maíz, seguida por el viaje en canoa hacia el inframundo, y culmina con su nacimiento.

En su argumento, Chinchilla equipara el ruido producido por la música y el baile del dios con el que produjeron los héroes gemelos del Popol Vuh mientras jugaban a la pelota, lo cual importunó a los señores de Xibalbá y motivó el viaje en canoa para enfrentarlos. El tema de las mujeres jugueteando en el agua resulta com-

${ }^{9}$ Generalmente estas deidades, a quienes Coe (1978: 12; 1989: 167) identificó como Headband Gods, o Dioses con diadema, han sido asociados con los héroes gemelos del Popol Vuh. Erik Velásquez (com. pers., 2013) nos indicó que el Dios Ch lleva por nombre Yaxh B'ahlun o Primer Nueve, mientras al Dios S se le conoce como Ju'n Ajaw o 1 Señor. Este último recibe asimismo el nombre de Ju'n Pu'w o 1 Cerbatana en los vasos K793 y K7727, lo que recuerda a Jun Ajpu' de los k'iche'. 
plicado. Siguiendo a Taube, el autor sugiere la posibilidad de que éstas sean consortes del Dios del maíz, departiendo lúbricamente en un espacio relacionado con la seducción; incluso establece la similitud de una de ellas con Xochiquetzal. También destaca que los rasgos de las doncellas expresan su pertenencia al inframundo, lo cual insinúa la muerte del Dios del maíz. Para reforzar su argumento alude al llamado Vaso de la Muerte ubicado en el Museo Etnográfico de Berlín, en cuya inscripción se menciona la muerte de un personaje histórico al que presumiblemente ataviaron con los atributos del dios.

No obstante, Chinchilla (op. cit.: 63) señala que Taube demostró que el vaso reitera la muerte del dios. La última escena en el Vaso de los Remeros es el nacimiento del Dios del maíz, que surge de las fauces de una serpiente. Para el autor, esta representación evoca algunos mitos documentados entre grupos ch'orti', popoluca y totonaca básicamente. Pone de manifiesto también aquellas representaciones donde la deidad sale de un cráneo hendido con plantas acuáticas, y alude al Plato Cósmico (K1609) en el que Chaahk quiebra el caparazón de la tortuga con su hacha, lo cual recuerda mitos contemporáneos donde un rayo desgaja la montaña que aloja los granos, aunque advierte que en estos últimos no se involucra al dios, sino que sólo se alude al hallazgo de los granos (Chinchilla, op. cit.: 81).

\section{Muerte y resurrección de J'un Imiix}

El tema del viaje en canoa fue abordado por Schele y Miller (1986: 271) a partir de cuatro huesos labrados que formaron parte del ajuar funerario de Jasaw Chan K'awiil. Una referencia previa corresponde a Kelley (1976: 236), quien destacó el carácter mítico de las escenas, subrayado por los animales que participan en la representación, ya que su presencia los vincula con la creación de los hombres de maíz, aunque también podría tratarse del nacimiento de la planta. En un trabajo posterior, Freidel, Schele y Parker (1999) establecieron la relación de la canoa con el mito de la creación. La equiparan con la Vía Láctea, cuyo movimiento aparente en la bóveda celeste parece el de precipitarse hacia el abismo. En ella, los Dioses remeros conducen al Dios del maíz hacia el lugar de la creación, un espacio situado entre Géminis y Orión, este último representado por el caparazón de una tortuga. Allí tuvo lugar el renacimiento del Dios del maíz, quien elevó el Árbol del Mundo, y se colocaron las tres piedras del fogón.

Encontramos convergencia entre diversos investigadores respecto a la naturaleza del viaje en canoa que realiza el Dios del maíz hacia el espacio primordial o bien al inframundo tras morir ritualmente, conducido por los Dioses remeros, psicopompos que eventualmente son sustituidos por los dioses Yaxh B'alun y Ju'n Ajaw (Ch y S). En su estudio sobre el Dios remero Jaguar y el Dios remero Espina de Raya, Velásquez (2010:116) señaló la asociación de ambas deidades con el nacimiento del maíz y su participación en momentos liminares como la creación 
del mundo, al ser ellos quienes asentaron una de las tres piedras fundacionales del fogón cósmico el hix tzam(?) tunnaj, el "trono de piedra jaguar", que colocaron en el Nah Ho' Chan o Quinto Cielo Primordial, su lugar de residencia. Velásquez también menciona el texto consignado en el vaso K688, el cual refiere que "en 13 Mak nació Jo..., ocurrió en Nah Ho' Chan Witz Xaman”, lo que sugiere que el maíz germinó en el corazón de la Montaña del Norte situada en aquel Quinto Cielo Primordial. Siguiendo a Martin, Velásquez asocia el nacimiento del Dios del maíz con la inmolación del Bebé Jaguar, 'Unen B'ahlam, quien es ejecutado por Yax Ha'al Chaak, el dios de las primeras lluvias, mientras danza. Esta escena se desarrolla precisamente en la Montaña del Norte del Nah Ho' Chan, donde el Dios de la muerte extiende sus brazos para recibir al sacrificado (figura 6).

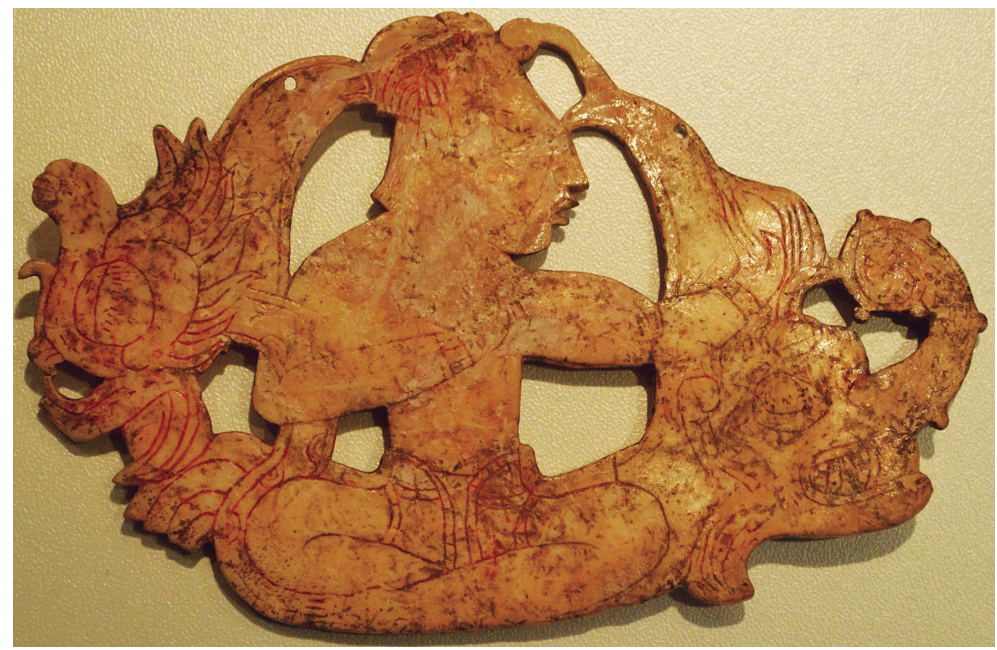

Figura 6. Placa de concha de Xcambó. Fotografía: D. Juárez.

Respecto a la identificación de las doncellas desnudas que ayudan al Dios del maíz a vestirse entre las aguas, consideramos que no parece haber elementos que sugieran su relación como consortes. Si suponemos que el dios viajó hacia el "lugar de la creación" o descendió al inframundo, y que las doncellas realmente lo están preparando para su eventual renacimiento que culminaría con una danza para expresar su triunfo sobre la muerte, cabría esperar entonces que la deidad se encontrase en ese momento en un espacio liminar al que fue transportado por los Dioses remeros. En su trabajo sobre los ritos de paso, Van Gennep (2008: 37) planteó que franquear el umbral tiene como propósito agregarse a un mundo diferente, de tal manera que las ceremonias y preparativos que se realizan en el umbral configuran ritos de margen. Las mujeres que vemos desnudas y en cuyos rostros Chinchilla identifica claramente párpados obscuros y el signo de "\%" en las 
mejillas, asociado con seres del inframundo, señalan sin lugar a duda que todas ellas pertenecen a ese ámbito. De tal manera que podría sugerirse que son una especie de guardianas del umbral. Un argumento en apoyo a esta idea parece insinuarse en el vaso K511 del University Art Museum de Princeton que se publicó en el catálogo The Blood of Kings (Schele y Miller, 1986: 297, pl. 115a). La escena tiene lugar en el Lago Negro, el inframundo, como lo atestigua el Dios L desde su sitial, quien es atendido por cinco doncellas engalanadas con sutiles enredos de gasa; parece indudable que forman parte de ese mundo. En la topografía de la cosmovisión maya, la tierra y el inframundo estaban separados por las aguas primordiales, de tal manera que las doncellas también alcanzan este último ámbito liminar.

Por otra parte, no debemos perder de vista el papel que desempeña el agua en las ceremonias de purificación que Van Gennep caracteriza como ritos de separación. Destaca el autor que en ellos el sujeto no debe tocar la tierra, puesto que en ese momento no pertenece al mundo sagrado ni al profano: transgredir esta observación frenaría inoportunamente la separación del mundo que se pretende abandonar. Conviene agregar también que los ritos de separación implican la idea de vestirse por vez primera (Van Gennep, op. cit.: 84).

Hay una imagen del mundo helénico hábilmente retratada por Karl Kerényi (2010: 66) que recuerda escenas similares, cuando se refiere a las ninfas que habitan en los manantiales, cuyo papel es el de niñeras que cuidan de los infantes divinos y semidioses, con quienes bailan, las cuales de alguna manera simbolizan la exuberante fecundidad. Quizá como parte de este rico imaginario en el que participan doncellas, valdría la pena incluir el hallazgo relativamente reciente de seis figurillas femeninas en una tumba de Tak'alik Ab'aj. En efecto, en 2012, Shieber y Orrego (2013) descubrieron la tumba de un personaje a quien denominaron K'utz Cham o el Abuelo Buitre, la cual fecharon alrededor del año 500 a.C. Entre los objetos que fueron depositados en su interior como parte del ajuar funerario destacan seis figurillas de mujeres desnudas delicadamente elaboradas en barro. Quizás ésta podría ser una de las referencias más tempranas que se conservan sobre las doncellas vinculadas con el inframundo, referencia que acaso también nos ayude a comprender mejor su relación con el Dios del maíz, los gobernantes y la participación que tuvieron en ese renacer al mundo.

Tras el baño lustral, el Dios del maíz recibe su nueva indumentaria: está preparado para retoñar. Como parte de un ritual iniciático, Thomas (1983: 530) señaló que esta parte del proceso se percibe como símbolo de la pureza rencontrada que preludia el triunfo sobre la muerte. La montaña floreciente se convierte en referente del mundo salvaje, del indistinto primordial que ahora se constituye en escenario de la hierofanía que está por ocurrir: el renacimiento del dios como el brote de una planta de maíz. Este drama sugiere un paralelismo con la trama que sigue el misterio eleusino, también de carácter agrícola, que ha sido descrito por Karl Kerényi (2004). La muerte de la deidad es mítica y, por lo tanto, prolongación de la vida en sus dos dimensiones, una como planta y la otra divina. De tal manera que el sufrimiento y la muerte del ser divino propician que fluyan los bienes hacia la comunidad. 
La gran mayoría de las escenas que se conservan y aluden al renacimiento del Dios del maíz — desde los murales del Preclásico Tardío descubiertos en San Bartolo en el Petén hasta los platos polícromos del Clásico Tardío- lo muestran asistido por los dioses Yahx B'alun y Ju'n Ajaw, danzando y eventualmente acompañado por un ave acuática. Hay cierto consenso entre los investigadores estadounidenses en identificar a estos como los gemelos divinos del Popol Vuh, cuyo padre, Hun Hunahpú, es el Dios del maíz (Miller y Martin, 2004: 56). A Hunahpú se le reconoce por los puntos en las mejillas, y a Xbalanqué por los parches de piel de jaguar. Braakhuis (2009) ha cuestionado seriamente la identificación de Hun Hunahpú como el Dios del maíz, a cuyo referente en el calendario k'iche' se asocia con los ancestros, la muerte y los entierros.

Pero, más allá de esta discusión sobre a la identidad del Dios del maíz, el aspecto más interesante a destacar es el tema de la danza. Reents-Budet (1994: 179) ha identificado, en lo que denomina como Estilo Regional de Holmul, el tema del danzante que caracteriza a dicha cerámica, producida al parecer en los sitios de Holmul, Naranjo y Buenavista del Cayo, los dos primeros en el Petén y el último en Belice. Las representaciones aluden a gobernantes o principales personificando al Dios del maíz mientras realiza su danza triunfal acompañado por un cormorán o eventualmente un enano. Al parecer estas piezas tenían como destino el ajuar funerario de miembros de uno de los segmentos menos encumbrados de la nobleza.

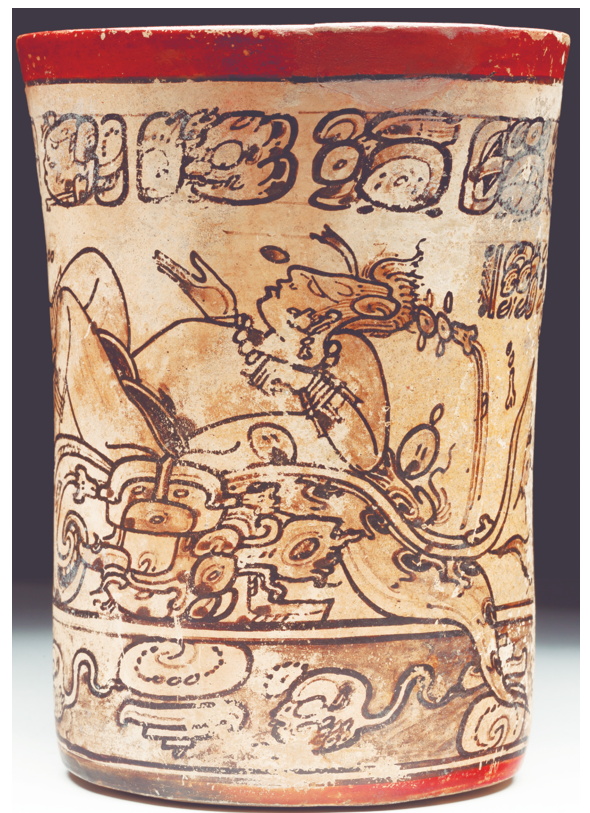

Figura 7. Vaso de Calakmul con la representación de 'Unen B'ahlam.

Museo Nacional de Antropología, No. de catálogo 05.0-04438. Fotografía: Archivo Digital. 
Los gobernantes mayas de las Tierras Bajas se hicieron representar adoptando el aspecto e indumentaria del Dios del maíz y ejecutando una danza ritual para reactualizar la creación del mundo. Era ésta la manera de participar en su renovación, actuando como mediadores de las deidades. Entre los tz'utujiles de Santiago Atitlán, Christenson ha documentado el baile ritual celebrado por el nab'eysil, uno de los sacerdote más importantes para la comunidad y quien considera que los ancestros actúan por su mediación. El eje de la celebración es una deidad identificada como Martín, representada por un atado de tela llamado ruk'ux way, ruk'ux ya', “corazón del alimento maíz, corazón de agua”, que tiene el poder para crear y mantener la vida como numen del maíz. Para el nab'eysil, nos dice Christenson, "la danza no es un símbolo de creación, sino un medio de regresar al amanecer del tiempo mismo, con el propósito de repetir las acciones de antecesores deificados y dioses" (2005: 94).

\section{Discusión y resultados}

Sin lugar a dudas, el pendiente que se exhibe en la Sala Maya del Museo Nacional de Antropología constituye una singular alegoría sobre el nacimiento del joven Dios del maíz como metáfora del ciclo agrícola. Hemos esbozado aquellos escenarios, elementos y personajes que participan de su integración iconográfica, utilizando para ello sólo una reducida muestra de las representaciones que configuraron el ciclo mítico. Si bien nuestro propósito no ha sido develar esta compleja saga que permeó en el imaginario de las élites mayas de la antiguiedad clásica, la identificación de las técnicas de manufactura mediante las cuales se confeccionó esta pieza destaca la relevancia que tuvo el mito para recrearlo en diferentes tipos de artefactos y especialmente en conchas, formalizado en los valores asignados a la elección de la materia prima y la habilidad de sus artistas para elaborarla. Por otra parte, resulta lamentable que no conozcamos las características de su contexto deposicional, pues éste seguramente habría aportado mayores elementos para su análisis.

Entre los numerosos objetos recuperados en Jaina y que forman parte del acervo de la colección maya del Museo Nacional de Antropología, alrededor de 6750 son de concha, y prácticamente todos corresponden a objetos terminados, que van desde cuentas hasta elaborados pendientes como el que nos ocupa. Hasta el momento, no tenemos evidencia de que la isla contara con talleres especializados para su manufactura. Benavides ha planteado que tanto Jaina como Isla Piedras y Uaymil son islas construidas artificialmente sobre la costa de Campeche. Bajo el control de las élites, funcionaron como puertos de embarque y transbordo, integrando una amplia red comercial que conectaba varias redes fluviales que desembocaban en el Golfo de México, así como sitios costeros de la Península de Yucatán. El análisis del material cerámico indica que el $60 \%$ de la cerámica fue elaborada regionalmente, un $20 \%$ proviene del centro de Veracruz y las Tierras Ba- 
jas noroccidentales, y el otro $20 \%$ del área comprendida entre Edzná y Calakmul (Benavides, 2012: 272). Como parte de este intercambio, a la isla llegaban, entre otros productos, basalto, cinabrio y jadeíta.

Conviene agregar que, a juzgar por los análisis de concha que hemos realizado en otros sitios como Calakmul, Moral-Reforma, Yaxchilán y Xuenkal, y por la identificación que hemos hecho de sus técnicas de manufactura, éstas corresponden con las características del utillaje lítico hallado en otros sitios del área maya para el trabajo de la concha durante los períodos Preclásico, Clásico y Posclásico. ${ }^{10}$ Esto desafortunadamente no es suficiente como para sugerir la ubicación de talleres especializados en su manufactura. Pese a ello, podemos plantear que estas piezas fueron elaboradas por artistas que formaban parte de las élites, cuyos talleres se encontraban dentro de ciertos sectores en los palacios, como lo han sugerido McAnany y Plank (2001: 94), al igual que Arlen y Diane Z. Chase (2001: 134).

Es de interés comentar, finalmente, que hay una perfecta correspondencia con las singulares piezas estudiadas por Velázquez en Dumbarton Oaks. El hecho que incluso compartan detalles como la elaboración de los calados mediante perforaciones hace factible proponer que son resultado de un mismo estilo tecnológico e iconográfico.

\section{Agradecimientos}

A Erik Velásquez por la lectura crítica del texto. Sus comentarios y observaciones enriquecieron sensiblemente nuestra perspectiva epigráfica e iconográfica sobre el Dios del maíz. A Antonio Benavides, cuyo conocimiento de Jaina nos permitió aproximarnos a las características contextuales de la isla. Y, de manera muy especial, a Roberto Jiménez Ovando, quien, antes de abordar el cayuco guiado por los Dioses remeros en su camino hacia la fuente de agua oscura, 'och ' $u$ ch'en? $e k$ ' naab'?, tuvo la generosidad y paciencia de compartir con nosotros sus vivencias en Jaina durante la temporada de 1964.

Las fotografías del pendiente, así como la del vaso de Calakmul, fueron proporcionadas por el Archivo Digital de la Colecciones del Museo Nacional de Antropología, CONACULTA-INAH-Canon.

\footnotetext{
${ }^{10}$ El uso de rocas calizas como instrumentos de desgaste ha sido identificado en varios sitios del área maya de los períodos Preclásico, Clásico y Posclásico, como Moral-Reforma, Calakmul, Calica, Xuenkal, Kohunlich e Ichpaatun. Incisiones y cortes hechos con utensilios de obsidiana se han detectado en Palenque, Moral-Reforma, Yaxchilán, Calakmul, Xuenkal, Oxtankah e Ichpaatun (al respecto véase Alonso et al., 2013; Colón, 2007; Castillo y Páez, 2013; Filloy, 2014; Melgar, 2008; Reyes, 2013; Velázquez y Juárez, 2007 y Velázquez et al., 2013).
} 


\section{BIBLIOGRAFÍA}

Alonso, Alejandra et al.

2013 "Análisis de las técnicas de manufactura de los objetos de concha de Xuenkal, Yucatán", Técnicas analíticas aplicadas a la caracterización y producción de materiales arqueológicos en el área maya, pp. 109-134, Adrián Velázquez Castro y Lynneth S. Lowe (eds.). México: Universidad Nacional Autónoma de México, Instituto de Investigaciones Filológicas, Centro de Estudios Mayas (Serie Testimonios y Materiales Arqueológicos para el Estudio de la Cultura Maya, 4).

Baudez, Claude F. (coord.)

1983 Introducción a la arqueología de Copán, Honduras, 3 t. Tegucigalpa: Instituto Hondureño de Antropología e Historia.

Benavides, Antonio

2012 Jaina: ciudad, puerto y mercado. Campeche: Gobierno del Estado de Campeche.

Braakhuis, H. E. M.

2009 "The Tonsured Maize god and Chicome-Xochitl as Maize Bringers and Culture Heroes: A Gulf Coast Perspective", Wayeb Notes, 32: 1-38.

Castillo Velasco, Elba Adriana y Shiat Alejandra Páez Torres

2013 "Manufactura de los objetos de concha de Calica, Quintana Roo", Técnicas analíticas aplicadas a la caracterización y producción de materiales arqueológicos en el área maya, pp. 95-108, Adrián Velázquez Castro y Lynneth S. Lowe (eds.). México: Universidad Nacional Autónoma de México, Instituto de Investigaciones Filológicas, Centro de Estudios Mayas (Serie Testimonios y Materiales Arqueológicos para el Estudio de la Cultura Maya, 4).

Chase, Arlen F. y Diane Chase

2001 "The Royal Court of Caracol, Belize: Its Palaces and People", Royal Courts of the Ancient Maya, II, pp. 102-137, Takeshi Inomata y Stephen D. Houston (eds.). Oxford: Westview Press.

Chinchilla Mazariegos, Oswaldo

2011 Imágenes de la mitología maya. Guatemala: Universidad Francisco Marroquín, Museo Popol Vuh.

Christenson, Allen J.

2005 "Bailando en las huellas de los antepasados", Los mayas, señores de la creación, pp. 91-96, Virginia M. Fields y Dorie Reents-Budet (comps.). San Sebastián, España: Nerea.

Coe, Michael D.

1978 Lords of the Underworld. Princeton: Princeton University Press.

1989 "The Hero Twins: Myth and Image", The Maya Vase Book, I, pp. 161-184, Justin Kerr (ed.). New York: Kerr Asociates. 
Colón González, Marinés

2007 "Una nueva visión del género Spondylus en Calakmul: técnicas de manufactura”, Los moluscos arqueológicos, una visión del mundo maya, pp. 99-123, Adrián Velázquez Castro y Lynneth S. Lowe (eds.). México: Universidad Nacional Autónoma de México, Instituto de Investigaciones Filológicas, Centro de Estudios Mayas (Cuadernos del Centro de Estudios Mayas, 34).

Fash, William L.

1991 Scribes, Warriors and Kings. Londres: Thames and Hudson.

1996 "Historia de las investigaciones arqueológicas en las Ruinas de Copán", Visión del pasado maya, pp. 31-47, William L. Fash y Ricardo Agurcia Fasquelle (coords.). Tegucigalpa: Asociación Copán (Publicación 2).

Filloy Nadal, Laura

2014 Costume et insignes d'un gouvernant maya K'inich Janaab' Pakal de Palenque. Oxford: Archaeopress (BAR International Series, 2590).

Freidel, David, Linda Schele y Joy Parker

1999 El cosmos maya. México: Fondo de Cultura Económica.

García Campillo, José Miguel

1998 "Datos epigráficos para la historia de Jaina durante el período Clásico", Investigadores de la Cultura Maya, 6 (I), pp. 45-62. Campeche: Universidad Autónoma de Campeche.

García-Cubas, Antonio y Martha Regero

2004 Catálogo ilustrado de moluscos gasterópodos del Golfo de México y Mar Caribe. México: Universidad Nacional Autónoma de México.

Houston, Stephen D.

2010 "Living Waters and Wondrous Beasts", Fiery Pool. The Maya and the Mythic Sea, pp. 66-79, Daniel Finamore y Stephen D. Houston (eds.). Massachusetts: Peabody Essex Museum.

Kelley, David H.

1976 Deciphering the Maya Script. Austin: University of Texas Press.

Kerényi, Karl

2004 Eleusis. Madrid: Ediciones Siruela.

2010 Hermes, el conductor de almas. México: Sexto Piso.

McAnnany, Patricia A. y Shannon Plank

2001 "Perspectives on Actors, Gender Roles, and Architecture at Classic Maya Courts and Households", Royal Courts of the Ancient Maya, I, pp. 84-129, Takeshi Inomata y Stephen D. Houston (eds.). Oxford: Westview Press.

Melgar Tísoc, Emiliano Ricardo

2008 La explotación de recursos marino-litorales en Oxtankah. México: Instituto Nacional de Antropología e Historia (Serie Premios INAH). 
Miller, Mary E. y Simon Martin

2004 Courtly Art of the Ancient Maya. San Francisco: Thames and Hudson.

Pillsbury, Joanne et al. (eds.)

2012 Ancient Maya Art at Dumbarton Oaks. Washington, D.C.: Dumbarton Oaks Research Library and Collection.

Piña Chan, Román

1968 Jaina. La casa en el agua. México: Instituto Nacional de Antropología e Historia y Secretaría de Educación Pública.

Reents-Budet, Dorie

1994 Painting the Maya Universe: Royal Ceramics of the Classic Period. North Carolina Durham: Duke University Press.

Ricœur, Paul

1998 Teoría de la interpretación. Discurso y excedente de sentido. México: Siglo XXI Editores.

Sahlins, Marshall

2006 Cultura y razón práctica. Contra el utilitarismo en la teoría antropológica. Barcelona: Gedisa Editorial.

Schele, Linda y Mary Ellen Miller

1986 The Blood of Kings. Dynasty and Ritual in Maya Art. Forth Worth: Kimbell Art Museum.

Schieber de Lavarreda, Christa y Miguel Orrego Corzo

2013 “Descubriendo el Entierro Real No. 2 de Tak'alik Ab’aj”, Antropología e Historia de Guatemala, 3a época, 12: 7-33.

Schmidt, Peter, Mercedes de la Garza y Enrique Nalda (coords.)

1998 Los mayas. México: Universidad Nacional Autónoma de México, Instituto Nacional de Antropología e Historia y Consejo Nacional para la Cultura y las Artes.

Serrano Sánchez, Carlos y Sergio López Alonso

2007 "Estatus social y contexto funerario durante el Clásico en Jaina, Campeche", La población prehispánica de Jaina, pp. 77-110, Patricia Hernández y Lourdes Márquez (coords.). México: Instituto Nacional de Antropología e Historia, Escuela Nacional de Antropología e Historia y Consejo Nacional para la Cultura y las Artes.

Sierra Sosa, Thelma N.

2004 "La arqueología de Xcambó, Yucatán, centro administrativo salinero y puerto comercial de importancia regional durante el Clásico", tesis de doctorado en Estudios Mesoamericanos. México: Universidad Nacional Autónoma de México. 
Spinden, Herbert J.

1975A Study of Maya Art. Nueva York: Dover Publications, Inc.

Taube, Karl A.

1985 “Classic Maya Maize God: A Reappraisal”, Fifth Palenque Round Table, 1983, pp. 171-182, Virginia M. Fields (ed.). San Francisco: Pre-Columbian Art Research Institute.

Taylor, Dicey

1992 "Painted Ladies: Costumes for Womans on Tepeu Ceramics", The Maya Vase Book, 3, pp. 513-525, Justin Kerr (ed.). New York: Kerr Associates.

Thomas, Louis-Vincent

1983 Antropología de la muerte. México: Fondo de Cultura Económica.

Van Gennep, Arnold

2008 Los ritos de paso. Madrid: Alianza Editorial (Ciencias Sociales, 3020).

Velázquez Castro, Adrián

2007 La producción especializada de los objetos de concha del Templo Mayor de Tenochtitlan. México: Instituto Nacional de Antropología e Historia (Colección Científica, 519).

2012 "Pre-Columbian Maya Shell Objects: An Analysis of Manufacturing Techniques", Ancient Maya Art at Dumbarton Oaks, pp. 433-440, Joanne Pillsbury et al. (eds.). Washington: Dumbarton Oaks, Research, Library and Collection (Pre-Columbian Art at Dumbarton Oaks, 4).

Velázquez Castro, Adrián y Daniel Juárez Cossío

2007 "La colección de objetos de concha de Moral-Reforma", Los moluscos arqueológicos, pp. 61-97, Adrián Velázquez Castro y Lynneth S. Lowe (coords.). México: Universidad Nacional Autónoma de México, Instituto de Investigaciones Filológicas, Centro de Estudios Mayas (Cuadernos del Centro de Estudios Mayas, 34).

Velázquez Castro, Adrián et al.

2013 "Producción de artefactos de concha en la Pequeña Acrópolis de Yaxchilán", Técnicas analíticas aplicadas a la caracterización y producción de materiales arqueológicos en el área maya, pp. 31-65, Adrián Velázquez Castro y Lynneth S. Lowe (eds.). México: Universidad Nacional Autónoma de México, Instituto de Investigaciones Filológicas, Centro de Estudios Mayas (Serie Testimonios y Materiales Arqueológicos para el Estudio de la Cultura Maya, 4).

Velásquez García, Erik

2010 "Los Dioses remeros mayas y sus posibles contrapartes nahuas", The Maya and their Neighbors, pp. 115-131, Laura Van Broekhoven et al. (eds.). Germany, 10th European Maya Conference. Verlag: Anton Saurwin. 
